\title{
Neurocognitive Outcomes in the Treatment of Early-Onset Schizophrenia Spectrum Disorders Study
}

\author{
Dr. Jean A. Frazier, M.D., Dr. Anthony J. Giuliano, Ph.D., Dr. Jacqueline L. Johnson, Dr. \\ P.H., Ms. Lauren Yakutis, B.A., Dr. Eric A. Youngstrom, Ph.D., Dr. David Breiger, Ph.D., Dr. \\ Linmarie Sikich, M.D., Dr. Robert L. Findling, M.D. M.B.A., Dr. Jon McClellan, M.D., Dr. \\ Robert M. Hamer, Ph.D., Dr. Benedetto Vitiello, M.D., Dr. Jeffrey A. Lieberman, M.D., and Dr. \\ Stephen R. Hooper, Ph.D. \\ Dr. Frazier and Ms. Yakutis are with the University of Massachusetts Medical School, Worcester, \\ MA. Dr. Giuliano is with the Massachusetts Mental Health Center Public Psychiatry Division of the \\ Beth Israel Deaconess Medical Center. Drs. Johnson, Sikich, Hamer, Hooper, and Youngstrom \\ are with the University of North Carolina, Chapel Hill, NC. Dr. Findling is with Case Western \\ Reserve, Cleveland, OH. Drs. Breiger and McClellan are with the University of Washington, \\ Seattle, WA. Dr. Lieberman is with Columbia University College of Physicians and Surgeons. Dr. \\ Vitiello is with the National Institute of Mental Health, Bethesda, MD
}

(C2012 American Academy of Child \& Adolescent Psychiatry. Published by Elsevier Inc. All rights reserved

Correspondence to: Dr. Jean A. Frazier, University of Massachusetts Medical School, Biotech I, Suite 100, 365 Plantation St. Worcester, MA 01605, Phone: 508-856-5073/FAX: 508-856-8211, Jean.Frazier@umassmed.edu.

Drs. Frazier and Giuliano contributed equally to this article.

The following Research Associates contributed significantly to the data collection: Kathryn DiFrancesco, Case Western Reserve, Cleveland, OH; Leslie Pierson, University of Washington, Seattle, WA; Jennifer Varley, Andrew Petesch, Vicki Dixon, Johna Hughes, and Madeline Puglia, University of North Carolina, Chapel Hill, NC; Emily Gerstein and Molly Wood, Cambridge Health Alliance, Cambridge, MA.

The opinions and assertions contained here are the views of the authors and are not to be construed as official or as reflecting the views of the Department of Health and Human Services, the NIH, or the NIMH. Dr. Jacqueline L. Johnson was our statistical expert. Clinical trial registry information: Treatment of Early-Onset Schizophrenia Spectrum Disorders (TEOSS); http://www.clinicaltrials.gov; NCT00053703.

Disclosure: Dr. Frazier has received research support from or participated in clinical trials with Bristol-Myers Squibb, GlaxoSmithKline, Janssen, Johnson and Johnson, Neuropharm, Otsuka American, Seaside Therapeutics, and Pfizer. Dr. Findling has received research support from, served as a consultant for, received royalties from, and/or served on the speakers' bureau for Abbot, Adrenex, Alexza, American Psychiatric Press, AstraZeneca, Biovail, Bristol-Myers Squibb, Forest, GlaxoSmithKline, Guilford Press, Johns Hopkins University Press, Johnson and Johnson, KemPharm Lilly, Lundbeck, Merck, National Institutes of Health, Neuropharm, Novatris, Noven, Organon, Otsuka, Pfizer, Physicians' PostGraduate Press, Rhodes, Roche, Sage, Sanofi-Aventis, Schering-Plough, Seaside Therapeutics, Sepracore, Shionogi, Shire, Solvay, Stanley Medical Research Institute, Sunovion, Supernus, Transcept, Validus, WebMD, and Wyeth. Dr. Hamer has received research support, served as a consultant for, or served on a data safety monitoring board/independent data monitoring committee for Acadia, Allergan, Alpharma, AstraZeneca, Cenerx, Corcept, EnabledMD, Epix, Johnson and Johnson, Novartis, Pepper-Hamilton, Pfizer, SAS Institute, Schwartz, Solvey, Sonofi-Aventis, Takeda, Winston-Strawn (a law suit involving Forest, Lundbeck, Sun, and Caraco), and Wyeth. He and/or his spouse are stockholders in Bristol-Myers Squibb, Amgen, Eli Lilly and Co., Genentech, Proctor and Gamble, and Sepracor. Dr. Hooper has received research support from and served as a consultant for Eli Lilly and Co. Dr. Lieberman has served on the advisory boards for Bioline, Pierre Fabre, and PsychoGenics. He has received grant support from Allon, GlaxoSmithKline, Eli Lilly and Co., Intercellular Therapies, Merck, Novartis, Pfizer, F. Hoffman-La Roche, Pepracor (Sunovion), and Targacept. He holds a patent from Repligen. Dr. McClellan has received research support from Pfizer. Dr. Sikich has received research support from or participated in clinical trials with BristolMyers Squibb, Curemark, Pfizer, Otsuka, and Seaside Therapeutics. He has served as a consultant for Sanofi Aventis and ABT Associates. He has received software from Posit Science. Dr. Youngstrom has served on the data safety monitoring board for Eli Lilly and Co. He has served as a consultant for Otsuka. He has received travel support from Bristol-Myers Squibb. Drs. Breiger, Giuliano, Johnson, and Vitiello, and Ms. Yakutis report no biomedical financial interests or potential conflicts of interest.

Publisher's Disclaimer: This is a PDF file of an unedited manuscript that has been accepted for publication. As a service to our customers we are providing this early version of the manuscript. The manuscript will undergo copyediting, typesetting, and review of the resulting proof before it is published in its final citable form. Please note that during the production process errors may be discovered which could affect the content, and all legal disclaimers that apply to the journal pertain. 


\section{Abstract}

Objective-To assess neurocognitive outcomes following antipsychotic intervention in youth enrolled in the National Institute of Mental Health (NIMH)-funded Treatment of Early-Onset Schizophrenia Spectrum Disorders (TEOSS).

Method-Neurocognitive functioning of youth (ages 8-19 years) with schizophrenia or schizoaffective disorder was evaluated in a four-site randomized, double-blind clinical trial comparing molindone, olanzapine or risperidone. The primary outcomes were overall group change from baseline in neurocognitive composite and six domain scores after 8 weeks and continued treatment up to 52 weeks. Age and sex were included as covariates in all analyses.

Results-Seventy-seven of 116 TEOSS participants (66\%) had post-baseline neurocognitive data. No significant differences emerged in the neurocognitive outcomes of the three medication groups. Therefore, the three treatment groups were combined into one group to assess overall neurocognitive outcomes. Significant modest improvements were observed in the composite score and in three of six domain scores in the acute phase, and in four of six domain scores in the combined acute and maintenance phases. Partial correlation analyses revealed very few relationships among Positive and Negative Syndrome Scale (PANSS) baseline or change scores and neurocognition change scores.

Conclusions-Antipsychotic intervention in youth with early-onset schizophrenia spectrum disorders (EOSS) led to modest improvement in measures of neurocognitive function. The changes in cognition were largely unrelated to baseline symptoms or symptom change. Small treatment effect sizes, easily accounted for by practice effects, highlight the critical need for the development of more efficacious interventions for the enduring neurocognitive deficits seen in EOSS.

\section{Keywords}

early-onset; schizophrenia; neurocognition; outcomes; antipsychotics

\section{INTRODUCTION}

Early-onset schizophrenia (EOS) is defined by having the onset of symptoms prior to age 18. The lifetime prevalence of schizophrenia is $1 \%$, with a substantial number of individuals diagnosed prior to age 18. ${ }^{1,2}$ EOS is often more severe than adult-onset schizophrenia (AOS) and includes both a higher rate of premorbid abnormalities and worse functional outcome ${ }^{3-5}$ Studies have documented comparable neurocognitive deficits in EOS and AOS,${ }^{6,7}$ including a recent meta-analysis that showed mostly large deficits for EOS compared to controls [effect sizes ranged from -1.27 (processing speed) to -0.58 (motor skills)]. ${ }^{8}$ Another meta-analysis documented more severe neurocognitive deficits in EOS compared with AOS, ${ }^{9}$ suggesting that illness severity may be associated with age of onset. ${ }^{10}$ The adult literature demonstrates that neurocognitive deficits are stronger predictors of social and role functioning than other illness features. ${ }^{11,12}$ Similarly, a recent 13 -year follow-up study of EOS found that baseline neurocognitive measures were associated with social and community functioning at follow-up. ${ }^{13}$

Neurocognitive functioning is an important target of treatment. ${ }^{14}$ Much of the earlier research on antipsychotics in adults with schizophrenia suggested favorable cognitive enhancing effects of second-generation antipsychotics (SGAs) over first-generation antipsychotics (FGAs). ${ }^{15}$ Data from the NIMH-sponsored Clinical Antipsychotic Trials of Intervention Effectiveness (CATIE) ${ }^{16}$ and the European First Episode Schizophrenia Trial (EUFEST) randomized clinical trials, ${ }^{17}$ however, failed to support the early claims of SGA superiority. Both CATIE and EUFEST interpreted their lack of differential medication 
effects as being due, in part, to the administration of FGAs at lower and more appropriate doses. The effects of antipsychotics in youth with EOSS disorders are just beginning to be understood. Controlled efficacy studies ${ }^{18-23}$ have documented that antipsychotics are superior to placebo in reducing positive and negative symptoms, but there have been no studies of antipsychotic effects on cognition in youth with EOSS.

The NIMH-funded multisite randomized controlled trial "Treatment of Early-Onset Schizophrenia Spectrum Disorders (TEOSS)" provided an opportunity to examine this issue in the largest sample of youths with EOSS studied to date. ${ }^{4,24}$ The initial purpose of this study was to compare the effects of olanzapine, risperidone, and molindone on neurocognition. However, due to limited power, the main focus of this paper is on whole group analyses to evaluate neurocognitive outcomes following antipsychotic treatment. We expected that the overall group would demonstrate modest improvements in neurocognition with antipsychotic treatment as evidenced by change in a single composite score and, to a lesser degree, change in specific domain scores at 8 weeks and 52 weeks or termination. We also assessed relationships among baseline and changes in clinical symptoms and change in the neurocognitive composite and domain scores.

\section{METHOD}

\section{Study Design}

The TEOSS study design, methods and results have been described previously. ${ }^{20,} 22,24$ TEOSS participants were randomized to one of three active treatments (risperidone, olanzapine or molindone). In this report, we focus on treatment-related change in neurocognition from baseline to weeks 8 and 52/termination, and between weeks 8 and 52/ termination. Neurocognitive assessments were administered at baseline and the end of the acute phase (week 8). After acute treatment (week 8), participants who had adequate response (20\% decrease in baseline Positive and Negative Syndrome Scale [PANSS] ${ }^{25}$ score plus a Clinical Global Impression-Improvement $[\mathrm{CGI}-\mathrm{I}]^{26}$ score of $\leq 2$ ) and tolerated their randomized treatment, were eligible to begin maintenance phase treatment with the same antipsychotic medication under double-blind conditions. Individuals who did not have adequate response at week 8 , or relapsed or experienced intolerable adverse effects during either the acute or maintenance phase, were offered randomly assigned, double-blind treatment with one of the other study medications for up to 52 weeks of treatment. ${ }^{20}$ The data reported here are based on changes observed during treatment with whichever medication was used for the longest period. The maintenance phase was up to a total of 44 weeks and the TEOSS neurocognitive battery was administered a third time at the end of the maintenance phase or at study termination. Neurocognitive assessments were obtained at week 8 and 52/termination during all subsequent treatments.

\section{Participants}

TEOSS enrolled youths (age 8 to 19 years) meeting DSM-IV diagnostic criteria for schizophrenia, schizoaffective disorder or schizophreniform disorder. ${ }^{27}$ Diagnoses were confirmed with the Structured Clinical Interview for DSM-IV Childhood Diagnoses (KIDSCID) ${ }^{28}$ Participants also had to have a score of moderate or greater on at least one PANSS positive symptom item. ${ }^{29}$ Additional details regarding methods and baseline sample characteristics are described elsewhere. ${ }^{4,7,22,24}$

\section{Neurocognitive Measures}

The battery of tasks assessed the following neurocognitive domains: general intellectual functioning [Wechsler Abbreviated Scale of Intelligence (WASI) ${ }^{30}$; Academic achievement skills [Wide Range Achievement Test (WRAT-3) ${ }^{31}$ Reading, Spelling and Arithmetic 
subtests]; Fine-motor speed and coordination [Finger Tapping Test (FT), ${ }^{32}$ Grooved Pegboard (GP $)^{33}$; Attention and inhibitory control [auditory and visual versions of VIGIL Continuous Performance Test $(\mathrm{CPT})^{34}$ ]; Short-Term Memory [Hopkins Verbal Learning Test-Revised (HVLT-R), ${ }^{35}$ Wide Range Assessment of Memory and Learning (WRAML) ${ }^{36}$ Visual Learning subtest, Visuospatial Working Memory Test (VSWM), ${ }^{37}$ WoodcockJohnson-III (WJ-III) Numbers Reversed ${ }^{38}$ ]; Cognitive Efficiency [Controlled Oral Word Association Test (COWAT), ${ }^{39}$ Ruff Figural Fluency Test (RFFT), ${ }^{40}$; ; and Social Cognition [Eyes Test ${ }^{41}$ ]. The WASI, WRAT-3, and Wisconsin Card Sorting Test-64-Computer Version (WCST-64-CV) ${ }^{42}$ were administered at baseline and termination only; all other measures were administered at each time point. Cognitive measures were selected based on their established psychometric properties, potential sensitivity to medication treatment, and general alignment with CATIE. ${ }^{16}$

\section{Data Analysis}

Neurocognitive Dataset Construction-The baseline neurocognitive data always came from the week a patient entered TEOSS (week zero). That is, baseline data remained constant, regardless of re-randomization in order to insure a uniform starting point (i.e., study entry) for all subjects. In contrast, post-baseline data points varied to some extent across subjects. To minimize practice effects, all data from a post-baseline (e.g., "week 8") neurocognitive visit were excluded if the visit occurred less than six weeks after the previous visit. Data included for the week 8 and termination assessments came from weeks 6 through 10 and 16 through 52, respectively. Some patients had post-baseline neurocognitive data from more than one medication randomization. To maximize sample size, data from all available randomizations were considered, but each patient only contributed one set of postbaseline scores to the analyses. For example, if the first randomization included both week 8 and termination data then the first randomization would be included. In general, the randomization that had the most post-baseline data was selected for inclusion in the postbaseline analysis. A later randomization was included instead of an earlier one if the latter had both week 8 and termination data and the earlier randomization had only week 8 data or only termination data. In all cases, regardless of which randomization was chosen for inclusion in the post-baseline data set, the baseline data was always from week 0 .

\section{Data Reduction: Neurocognitive Domain and Composite Scores}

Given that most single neuropsychological measures are moderately intercorrelated, ${ }^{43,44}$ we chose to reduce the number of variables for analysis by constructing domain and composite score measures, a strategy commonly employed in clinical trials. ${ }^{16}$ Such summary measures are also often more sensitive to treatment effects than single test scores, and composite measures are often more reliable and sensitive than domain scores. ${ }^{45}$ Construction of domain scores was rationally derived and consistent with our earlier work. ${ }^{7}$ Each individual test measure was evaluated for normality and outliers to support subsequent data reduction. Single test measures were first converted to standardized $z$-scores by subtracting the baseline mean and dividing by the baseline standard deviation based on the sample contributing to the analysis of each test measure. Such standardization using baseline scores was performed to produce three sets of standardized single test measures, one for each of the groups of subjects contributing to the baseline to 8 weeks, 8 weeks to 52 weeks, and baseline to 52 weeks analyses of each test measure. Domain summary scores were then constructed by calculating the mean of the $z$-scores for the measures that comprised the domain, and then re-standardizing the domains using baseline data as described previously. This re-standardization was employed so that baseline data for each of the three time point analyses of each domain had a mean of 0 and a standard deviation of 1 , enabling postbaseline means to be interpreted as effect sizes. This process resulted in six domain scores as follows: Fine Motor Speed/Coordination; Attention; Short-Term Memory; Cognitive 
Efficiency; Inhibitory Control (auditory and visual Vigil CPT commission errors); and Social Cognition. Additionally, composite scores for Academic Achievement and full-scale IQ were constructed in a similar way. See Table 1 for a list of neurocognitive assessments included in each domain and composite.

An overall neurocognitive composite score was calculated as an unweighted average of the six domain summary scores: Fine Motor Speed/Coordination, Attention, Short-Term Memory, Cognitive Efficiency, Inhibitory Control, and Social Cognition. After averaging the six domain summary scores, the composite score was re-standardized using baseline data as described earlier, so that post-baseline means could be interpreted as effect sizes. A composite score was computed only for those subjects with available data on at least 4 of 6 of the domains $(\mathrm{n}=70)$. Full scale IQ and Academic Achievement summary scores were computed for baseline and week 52/termination and were not available for week 8 per the study design. They did not contribute to the neurocognitive composite score and are treated as composite scores themselves in the analysis. This resulted in a total of six domain and three composite scores for subsequent analyses. Note that data from the WCST-64-CV was not included in these analyses as data was only available for baseline and 52 week/ termination time points per the study design, and the goal of this study was to examine change across three time points (baseline, 8-week/acute phase, and 52-week/termination); as a result, the problem-solving efficiency domain name that we used in our baseline neurocognitive paper $^{7}$ was changed to cognitive efficiency here to better reflect the constituent measures of the domain. Four (multi-measure) domain reliability coefficients were acceptable (i.e., Cronbach alpha reliability coefficients ranged between 0.78 and 0.80 ); however, the internal consistency of the Problem-Solving Efficiency domain was 0.64.

\section{Statistical Methods}

Descriptive statistics for all neurocognitive domain and composite scores were examined for normality and outliers. Change in each standardized neurocognitive domain and composite score from baseline to week 8 or termination/endpoint was compared using multiple analysis of covariance (ANCOVA), adjusting for baseline score, age and sex. For clarity and consistency in interpreting the results, age and sex were included as covariates in all models regardless of whether either was a significant predictor in the model. Given multiple comparisons, a Bonferroni correction would establish conservative critical values of $p<$ 0.008 for the six neurocognitive domain variables $(0.05 / 6)$ and $p<0.017$ for the neurocognitive composite, IQ, and academic achievement summary scores $(0.05 / 3)$. Standardized mean change scores provide an effect size estimate that indexes the magnitude of the difference in cognitive domain performance level between time points; positive standardized mean change scores indicate performance improvement, while negative values suggest reduced performance. In order to assess whether there was any greater or lesser cognitive improvement in the younger children versus the older children, we inspected whether age was a significant predictor in the model for each neurocognitive outcome and time point comparison. To correct for the analysis of multiple outcomes, the same Bonferroni p-value corrections were applied to the hypothesis tests determining significance of age in each model. Lastly, partial correlation analyses (adjusted for age and sex) were conducted to examine relationships among PANSS (Total, Positive, and Negative scale) baseline and change scores and neurocognitive domain and composite change scores. Significance levels for the correlation analyses were set at $p<0.008$ for the six domain composite scores and $p<0.017$ for the three other composite scores. Analyses were conducted using SAS 9.2. 


\section{RESULTS}

\section{Subjects and Baseline Characteristics}

Of the 116 participants treated in TEOSS, $77(66 \%)$ had post-baseline neurocognitive data suitable for analysis (see Figure 1). Table 2 shows baseline demographic and clinical characteristics of the sample with post-baseline neurocognitive data and those without. The sample was $2 / 3$ male and, on average, $14.3 \pm 2.5$ years old. Youth included in these analyses did not differ significantly from the non-neurocognitive sample (those without baseline or post-baseline neurocognitive data; $n=39$ ) on most variables. However, the neurocognitive sample was more symptomatic at baseline than the non-neurocognitive sample, having higher PANSS-Total, PANSS-Negative, and BPRS-C Total Scores. Because baseline neurocognitive data was often not available for the non-neurocognitive sub-sample, Table 2 does not provide group comparative baseline neurocognitive data.

\section{Neurocognitive Effects of Treatment}

There were no significant differences among the three medication groups on any neurocognitive change variable at any time point. Given limited statistical power, the groups were combined to focus on change in neurocognition for the overall group. Table 3 presents the means and standard deviations for the neurocognitive variables at each assessment point, and their related mean standardized change score (and standard deviation) around which these results are organized. Note that sample sizes vary across neurocognitive variables based on available data, and are reduced when comparisons are made with the 52-week/ termination assessment point. Change in the neurocognitive composite score, the primary outcome measure, showed significant improvement during acute and combined acute and maintenance treatment. Compared to their study entry baseline performance, participants' neurocognitive composite showed modest improvement by week $8(z=0.27, p<.0001)$ and improvement at 52-weeks/termination $(z=0.31, p=0.0005)$. Significant differences were not observed on the composite score between the 8-week and 52-week/termination assessments.

Significant change was seen in three of six neurocognitive domains between baseline and week 8 and four of six domains between baseline and week 52/termination (see Table 2). Significant improvements were shown in Fine motor speed/coordination at 8-weeks ( $z=$ $0.21, p=0.0016)$ and 52-weeks/termination $(z=0.27, p=0.006)$. Inhibitory Control also showed significant improvements at both time points $(z=0.25, p<0.0001 ; z=0.25, p=$ 0.0007 , respectively). Significant improvements were documented in Social Cognition between baseline and 8-weeks ( $z=0.28, p=0.0072)$ only, and in Attention $(z=0.16, p=$ $0.0048)$ and Cognitive Efficiency $(z=0.28, p=0.0008)$ between baseline and 52-weeks/ termination only. One neurocognitive domain, Cognitive Efficiency, demonstrated improvement over the 8 to 52 weeks maintenance phase $(z=0.18, p=0.013)$. Notably, no significant changes were observed in the Short-Term Memory domain at any follow-up assessment point. All effect sizes were small to very small.

In the analysis of age as predictor in the model for each neurocognitive outcome and time point comparison, only the model for change in Attention between weeks 8 and termination was significant, indicating that older children showed greater improvement in attention than younger children (coefficient for the age predictor $=0.132 ; \mathrm{p}=0.0007$ ). No other significant differential age effects were found.

As expected, there was no significant change observed in the WASI Full Scale IQ $(z=0.06$, $p=0.33$ ) or in the Academic Achievement composite score based on the WRAT-3 subtests $(z=-0.04, p=0.51)$. 


\section{Neurocognitive Change and Baseline Symptom Severity and Symptom}

Change-Partial Pearson correlations (adjusting for age and sex) between change in neurocognitive composite and domain scores from baseline and clinical symptom severity at baseline and change in PANSS total score and symptom factors were calculated to examine their associations. Using criteria for significance of $p=0.008$ for the domains and 0.017 for the composites, few significant relationships emerged. The correlation between change in PANSS Total and change in Attention from 8 to 52 weeks was significant $(r=0.41, p=$ $0.006)$ and the correlation between baseline PANSS Negative and change in Fine Motor Speed from week 8 to 52 was significant $(r=-0.38, p=0.007)$. These data suggest little reliable relationship between baseline levels of symptoms or their change over time and change in any neurocognitive index.

\section{DISCUSSION}

This is the first study of EOS to prospectively assess neurocognitive functioning in a large, well-characterized sample of youth over the course of a 52-week double-blind treatment trial with antipsychotic medication. We found that neurocognition, as indexed by a neurocognitive composite score, improved modestly with antipsychotic treatment between study entry and 8- and 52-weeks, with the largest proportion of the small effect emerging in the acute phase of the study. There was no significant change in overall neurocognition between 8- and 52-weeks/termination. At the level of specific neuropsychological domains, very small to small changes in several domain scores achieved statistical significance. These included changes in the Fine Motor, Inhibitory Control, and Social Cognition domains between baseline and week-8, and Fine Motor, Inhibitory Control, Attention, and Cognitive Efficiency between baseline and week-52/termination. No significant changes were observed in Short-Term Memory (which included measures of new learning), nor, as expected, were changes observed in Full Scale IQ or the Academic Achievement composite score at any time point. With the exception of greater improvements in attention from week 8 to termination in the older youth, age was not a significant predictor of neurocognitive outcomes. In addition, although sample sizes are small, no differences among medication groups on neurocognitive outcomes were observed. Lastly, neurocognitive change at any time point was not reliably associated with baseline or change in diagnostic-clinical symptoms.

Our findings are consistent with recent large studies of adults in documenting minimal cognitive benefits with antipsychotic treatment. ${ }^{16,17,46}$ The small magnitude of the performance improvements that emerged in this trial and others have been interpreted as consistent with practice effects. ${ }^{16,45,47,48}$ Moreover, given that treatment effects were small and most likely to be observed in the acute phase, it is possible that practice and/or placebo effects were responsible for the changes in neurocognitive performance that emerged. ${ }^{47,48}$ Practice effects cannot be ruled out in the present study due to the absence of a control group. Several studies have estimated that practice effects are at least in the small and often in the moderate range, including for tests similar to those used in TEOSS (e.g., MATRICS). ${ }^{49}$ Some clinical treatment study researchers have argued that when taking confidence intervals into consideration, effect sizes for conclusively nonrandom changes would have to be substantially higher. ${ }^{48}$

The absence of a reliable relationship between level of baseline clinical symptoms and neurocognitive change is not surprising given the ample literature documenting negligible to small relationships between clinical symptoms of schizophrenia and neurocognitive test scores in adults. ${ }^{7,} 50$ Similar findings are also noted in studies examining the relationship between cognitive change from baseline and changes in symptom measures. ${ }^{16}$ Nonetheless, symptom reduction might be associated with better task engagement which may, in turn, 
significantly change performance on cognitive measures. ${ }^{50}$ Despite treatment with antipsychotic medication, which substantially reduced clinical symptoms in approximately half of the TEOSS youth, ${ }^{22}$ cognitive symptoms remain an area of ongoing vulnerability that requires intervention and support across settings. At present, it appears that reducing symptoms of psychosis with medication is not sufficient for remedying cognitive deficits. However, it is reasonable to consider that by improving psychosis, antipsychotics might mitigate some of the psychosis associated cognitive deficits and prevent further decline.

Previous speculation about the benefits of antipsychotic medication on neurocognition has suggested that individuals with schizophrenia who are younger and closer to the onset of their symptoms, may be more likely to benefit from the cognitive enhancing effects of treatment. ${ }^{17,51}$ Unfortunately, our findings in this younger cohort do not support this hypothesis and are comparable to adult studies that included older individuals with chronic illness and more exposures to treatment and/or substances.

Others suggest that the cognitive enhancing effects of antipsychotic medication might be realized in the context of adequate environmental and cognitive stimulation. ${ }^{52}$ The premise is that just as muscle enhancing agents have little impact without exercise, so too might antipsychotic medication require routine environmental, cognitive, and/or behavioral stimulation to achieve a procognitive effect. In this vein, the majority of TEOSS subjects were in school, including $45 \%$ who were receiving special education at the time of enrollment. While TEOSS was not designed to track specific educational programming and interventions, the fact that nearly all TEOSS subjects were receiving some form of education-related cognitive stimulation tempers speculation that normative age-related cognitive stimulation might interact with medication treatment to yield procognitive benefits.

In addition to the absence of a control or placebo group, three limitations to the present study also require consideration. First, the majority of TEOSS participants had prior exposure to antipsychotics, ${ }^{22}$ thus raising the possibility that any potential cognitive enhancing effects of antipsychotic medication may have already been achieved (e.g., carryover effects from prior medication treatments) and was represented in their initial, baseline performance. Ethical concerns precluded a medication washout period and there was no medication-free assessment of neurocognitive function. Secondly, as is common in treatment studies, fewer patients contributed both baseline and follow-up neurocognitive data leaving a significant minority (34\%) who did not contribute data, thus limiting the generalizability of the findings to patients treated with antipsychotics in the community. Thirdly, we were unable to achieve the sample size and statistical power necessary to conduct strong comparative tests of potential differential medication effects on cognition. Low statistical power (which would allow detection of only large effects), however, limits the value of null findings in this regard. At the same time, between group effects sizes were negligible, and our findings complement recent large studies that have found no evidence of differential beneficial effects of second-generation antipsychotics over first-generation antipsychotics.

Youth in TEOSS demonstrated minimal neurocognitive improvement, and the improvements observed were consistent with practice effects as described in adults with chronic schizophrenia treated with first-generation antipsychotics and second-generation antipsychotics. Improvements in cognitive symptoms are much more difficult to achieve with either first-or second-generation antipsychotics than improvements in psychotic symptoms. Moreover, despite earlier and less methodologically rigorous research suggesting that second-generation antipsychotics provide broader clinical and cognitive benefit, TEOSS and related adult studies indicate that antipsychotic treatment selection cannot be based on 
the presumption of a differential cognitive benefit of one medication over another. Clinicians should not expect that all aspects of schizophrenia can be treated with a single intervention, including antipsychotics. While it is reasonable to hypothesize that antipsychotics might directly or indirectly mitigate psychosis-associated cognitive deficits, their impact on cognition is not sufficient to remedy functional deficits associated with the illness. Our findings underscore that treatment strategies other than antipsychotic medication are required to improve the cognitive functioning of youth with EOS. Given that cognitive deficits in EOS remain central, enduring, and functionally relevant, there remains a great need for augmentative and multi-modal treatments targeting cognition. These include the discovery of cognitive-enhancing agents and use of currently available psychosocial interventions (e.g., cognitive remediation ${ }^{53}$ Cognitive Adaptation Therapy ${ }^{54}$ and supported education interventions). Our data highlight the critical need for the development of effective interventions for the durable cognitive deficits associated with schizophrenia, particularly for youth with EOS given their cognitive and psychosocial at-risk status.

\section{Acknowledgments}

Treatment of Early-Onset Schizophrenia Spectrum Disorders (TEOSS) is funded by the National Institute of Mental Health (NIMH) under cooperative agreements U01MH61528 (LS), U01MH61464 (JM), U01MH62726 (JAF), and U01MH61355 (RLF), the Maternal Child Health Bureau (\#MCJ379154A), and the Administration on Developmental Disabilities (\#90DD043003). The research was conducted in National Institute of Health (NIH) supported Clinical Research Centers at University of Washington (M01-RR00037) and the University of North Carolina (MH01-RR00046). Study drugs for TEOSS were supplied by Janssen and Eli Lilly and Co.

\section{References}

1. Hafner H, Nowotny B. Epidemiology of early-onset schizophrenia. Eur Arch Psychiatry Clin Neurosci. 1995; 245(2):80-92. [PubMed: 7654792]

2. Thomsen PH. Schizophrenia with childhood and adolescent onset--a nationwide register-based study. Acta Psychiatr Scand. Sep; 1996 94(3):187-193. [PubMed: 8891086]

3. Eggers C, Bunk D. The long-term course of childhood-onset schizophrenia: a 42-year followup. Schizophr Bull. 1997; 23(1):105-117. [PubMed: 9050117]

4. Frazier JA, McClellan J, Findling RL, et al. Treatment of early-onset schizophrenia spectrum disorders (TEOSS): demographic and clinical characteristics. J Am Acad Child Adolesc Psychiatry. Aug; 2007 46(8):979-988. [PubMed: 17667477]

5. Rhinewine JP, Lencz T, Thaden EP, et al. Neurocognitive profile in adolescents with early-onset schizophrenia: clinical correlates. Biol Psychiatry. Nov; 2005 58(9):705-712. [PubMed: 16023084]

6. Holmen A, Juuhl-Langseth M, Thormodsen R, Melle I, Rund BR. Neuropsychological profile in early-onset schizophrenia-spectrum disorders: measured with the MATRICS battery. Schizophr Bull. Jul; 2010 36(4):852-859. [PubMed: 19223656]

7. Hooper SR, Giuliano AJ, Youngstrom EA, et al. Neurocognition in early-onset schizophrenia and schizoaffective disorders. J Am Acad Child Adolesc Psychiatry. Jan; 2010 49(1):52-60. [PubMed: 20215926]

8. Nieto RG, Castellanos FX. A meta-analysis of neuropsychological functioning in patients with early onset schizophrenia and pediatric bipolar disorder. J Clin Child Adolesc Psychol. 2011; 40(2):266280. [PubMed: 21391023]

9. Rajji TK, Ismail Z, Mulsant BH. Age at onset and cognition in schizophrenia: meta-analysis. Br J Psychiatry. Oct; 2009 195(4):286-293. [PubMed: 19794194]

10. DeLisi LE. The significance of age of onset for schizophrenia. Schizophr Bull. 1992; 18(2):209215. [PubMed: 1377833]

11. Green MF, Kern RS, Braff DL, Mintz J. Neurocognitive deficits and functional outcome in schizophrenia: are we measuring the "right stuff"? Schizophr Bull. 2000; 26(1):119-136. [PubMed: 10755673] 
12. Mohamed S, Rosenheck R, Swartz M, Stroup S, Lieberman JA, Keefe RS. Relationship of cognition and psychopathology to functional impairment in schizophrenia. Am J Psychiatry. Aug; 2008 165(8):978-987. [PubMed: 18450928]

13. Oie M, Sundet K, Ueland T. Neurocognition and functional outcome in early-onset schizophrenia and attention-deficit/hyperactivity disorder: a 13-year follow-up. Neuropsychology. Jan; 2011 25(1):25-35. [PubMed: 21090901]

14. Hyman SE, Fenton WS. Medicine. What are the right targets for psychopharmacology? Science. Jan; 2003 299(5605):350-351. [PubMed: 12532001]

15. Keefe RS, Silva SG, Perkins DO, Lieberman JA. The effects of atypical antipsychotic drugs on neurocognitive impairment in schizophrenia: a review and meta-analysis. Schizophr Bull. 1999; 25(2):201-222. [PubMed: 10416727]

16. Keefe RS, Bilder RM, Davis SM, et al. Neurocognitive effects of antipsychotic medications in patients with chronic schizophrenia in the CATIE Trial. Arch Gen Psychiatry. Jun; 2007 64(6): 633-647. [PubMed: 17548746]

17. Davidson M, Galderisi S, Weiser M, et al. Cognitive effects of antipsychotic drugs in first-episode schizophrenia and schizophreniform disorder: a randomized, open-label clinical trial (EUFEST). Am J Psychiatry. Jun; 2009 166(6):675-682. [PubMed: 19369319]

18. Kryzhanovskaya L, Schulz SC, McDougle C, et al. Olanzapine versus placebo in adolescents with schizophrenia: a 6-week, randomized, double-blind, placebo-controlled trial. J Am Acad Child Adolesc Psychiatry. Jan; 2009 48(1):60-70. [PubMed: 19057413]

19. Kumra S, Frazier JA, Jacobsen LK, et al. Childhood-onset schizophrenia. A double-blind clozapine-haloperidol comparison. Arch Gen Psychiatry. Dec; 1996 53(12):1090-1097. [PubMed: 8956674]

20. Findling RL, Johnson JL, McClellan J, et al. Double-blind maintenance safety and effectiveness findings from the Treatment of Early-Onset Schizophrenia Spectrum (TEOSS) study. J Am Acad Child Adolesc Psychiatry. Jun; 2010 49(6):583-594. quiz 632. [PubMed: 20494268]

21. Shaw P, Sporn A, Gogtay N, et al. Childhood-onset schizophrenia: A double-blind, randomized clozapine-olanzapine comparison. Arch Gen Psychiatry. Jul; 2006 63(7):721-730. [PubMed: 16818861]

22. Sikich L, Frazier JA, McClellan J, et al. Double-blind comparison of first- and second-generation antipsychotics in early-onset schizophrenia and schizo-affective disorder: findings from the treatment of early-onset schizophrenia spectrum disorders (TEOSS) study. Am J Psychiatry. Nov; 2008 165(11):1420-1431. [PubMed: 18794207]

23. Findling RL, Robb A, Nyilas M, et al. A multiple-center, randomized, double-blind, placebocontrolled study of oral aripiprazole for treatment of adolescents with schizophrenia. Am J Psychiatry. Nov; 2008 165(11):1432-1441. [PubMed: 18765484]

24. McClellan J, Sikich L, Findling RL, et al. Treatment of early-onset schizophrenia spectrum disorders (TEOSS): rationale, design, and methods. J Am Acad Child Adolesc Psychiatry. Aug; 2007 46(8):969-978. [PubMed: 17667476]

25. Kay SR, Opler LA, Lindenmayer JP. The Positive and Negative Syndrome Scale (PANSS): rationale and standardisation. Br J Psychiatry Suppl. Nov.1989 7:59-67. [PubMed: 2619982]

26. Guy, W. ECDEU Assessment Manual for Psychopharmacology. Washington, DC: National Institute of Mental Health; 1976. Clinical Global Impression; p. 217-221.

27. American Psychiatric Association. Diagnostic and Statistical Manual of Mental Disorders. 4. Washington, D.C: American Psychiatric Association; 1994.

28. Hein, D.; Matzner, F.; First, M.; Spitzer, R.; Williams, J.; Gibbon, M. Structured Clinical Interview for DSM-IV Childhoood Diagnoses, KID-SCID. New York: Columbia University Medical School; 1998.

29. Kay S, Fiszbein A, Opler LA. The positive and negative syndrome scale (PANNS) for schizophrenia. Schizophr Bull. 1987; 13:262-276.

30. Wechsler, D. Wechsler Abbreviated Scale of Intelligence (WASI). San Antonio, TX: Psychological Corporation; 1999.

31. Wilkenson, G. Wide Range Achievement Test- Third Revision. Wilmington, DE: Jastak Associates; 1993. 
32. Reitan, R.; Wolfson, D. The Halstead-Reitan Neuropsychological Test Battery: Theory of interpretation. Tuscon, AZ: Neuropsychology Press; 1985.

33. Lafayette Instrument Company. Manual for the Grooved Pegboard. Lafayette, IN: 1989.

34. Cegalis, J.; Bowlin, J. Vigil: Software for the Assessment of Attention. Nashua, NH: Forthought; 1991.

35. Brandt, J.; Benedict, R. The Hopkins Verbal Learning Test- Revised: Professional Manual. Odessa, FL: Psychological Assessment Resources Inc; 2001.

36. Sheslow, D.; Adams, W. Wide Range Assessment of Memory and Learning. Wilmington, DE: Jastak Associates; 1990.

37. Hershey T, Selke G, Fucetola R, Newcomer J. Spatial long-term memory but not working memory decreases over time in schizophrenia [Abstract]. Society for neuroscience abstracts. 1999; 25:572.

38. Woodcock, R.; McGrew, K.; Mather, N. Woodcock-Johnson III Tests of Cognitive Abilities. Itasca, IL: Riverside Publishing; 2001.

39. Benton, A.; Hamsher, K. Multilingual aphasia examination. Iowa City, IA: University of Iowa Press; 1978.

40. Ruff RM, Light RH, Evans RW. The ruff figural fluency test: A normative study with adults. Developmental Neuropsychology. 1987; 3(1):37- 51.

41. Baron-Cohen S, Wheelwright S, Hill J, Raste Y, Plumb I. The "Reading the Mind in the Eyes" Test revised version: a study with normal adults, and adults with Asperger syndrome or highfunctioning autism. J Child Psychol Psychiatry. Feb; 2001 42(2):241-251. [PubMed: 11280420]

42. Heaton, R.; Chelune, G.; Talley, J.; Kay, G.; Curtiss, G. Wisconsin Card Sorting Test manual: Revised and expanded. Odessa, FL: Psychological Assessment Resources; 1993.

43. Dickinson D, Gold JM. Less unique variance than meets the eye: overlap among traditional neuropsychological dimensions in schizophrenia. Schizophr Bull. May; 2008 34(3):423-434. [PubMed: 17702991]

44. Keefe RS, Bilder RM, Harvey PD, et al. Baseline neurocognitive deficits in the CATIE schizophrenia trial. Neuropsychopharmacology. Sep; 2006 31(9):2033-2046. [PubMed: 16641947]

45. Nuechterlein KH, Green MF, Kern RS, et al. The MATRICS Consensus Cognitive Battery, part 1: test selection, reliability, and validity. Am J Psychiatry. Feb; 2008 165(2):203-213. [PubMed: 18172019]

46. Wittorf A, Sickinger S, Wiedemann G, Klingberg S. Neurocognitive effects of atypical and conventional antipsychotic drugs in schizophrenia: a naturalistic 6-month follow-up study. Arch Clin Neuropsychol. May; 2008 23(3):271-282. [PubMed: 18272323]

47. Goldberg TE, Goldman RS, Burdick KE, et al. Cognitive improvement after treatment with second-generation antipsychotic medications in first-episode schizophrenia: is it a practice effect? Arch Gen Psychiatry. Oct; 2007 64(10):1115-1122. [PubMed: 17909123]

48. Goldberg TE, Keefe RS, Goldman RS, Robinson DG, Harvey PD. Circumstances under which practice does not make perfect: a review of the practice effect literature in schizophrenia and its relevance to clinical treatment studies. Neuropsychopharmacology. Apr; 2010 35(5):1053-1062. [PubMed: 20090669]

49. Keefe RS, Fox KH, Harvey PD, Cucchiaro J, Siu C, Loebel A. Characteristics of the MATRICS Consensus Cognitive Battery in a 29-site antipsychotic schizophrenia clinical trial. Schizophr Res. Feb; 2011 125(2-3):161-168. [PubMed: 21075600]

50. Bilder RM, Goldman RS, Robinson D, et al. Neuropsychology of first-episode schizophrenia: initial characterization and clinical correlates. Am J Psychiatry. Apr; 2000 157(4):549-559. [PubMed: 10739413]

51. Salimi K, Jarskog LF, Lieberman JA. Antipsychotic drugs for first-episode schizophrenia: a comparative review. CNS Drugs. Oct; 2009 23(10):837-855. [PubMed: 19739694]

52. Keefe RS, Vinogradov S, Medalia A, et al. Report from the working group conference on multisite trial design for cognitive remediation in schizophrenia. Schizophr Bull. Sep; 2011 37(5):10571065. [PubMed: 20194249]

53. Medalia, A.; Revheim, N.; Herlands, T. Cognitive remediation for psychological disorders, therapist guide. New York, NY: Oxford University Press; 2009. 
54. Velligan DI, Prihoda TJ, Ritch JL, Maples N, Bow-Thomas CC, Dassori A. A randomized singleblind pilot study of compensatory strategies in schizophrenia outpatients. Schizophr Bull. 2002; 28(2):283-292. [PubMed: 12693434] 


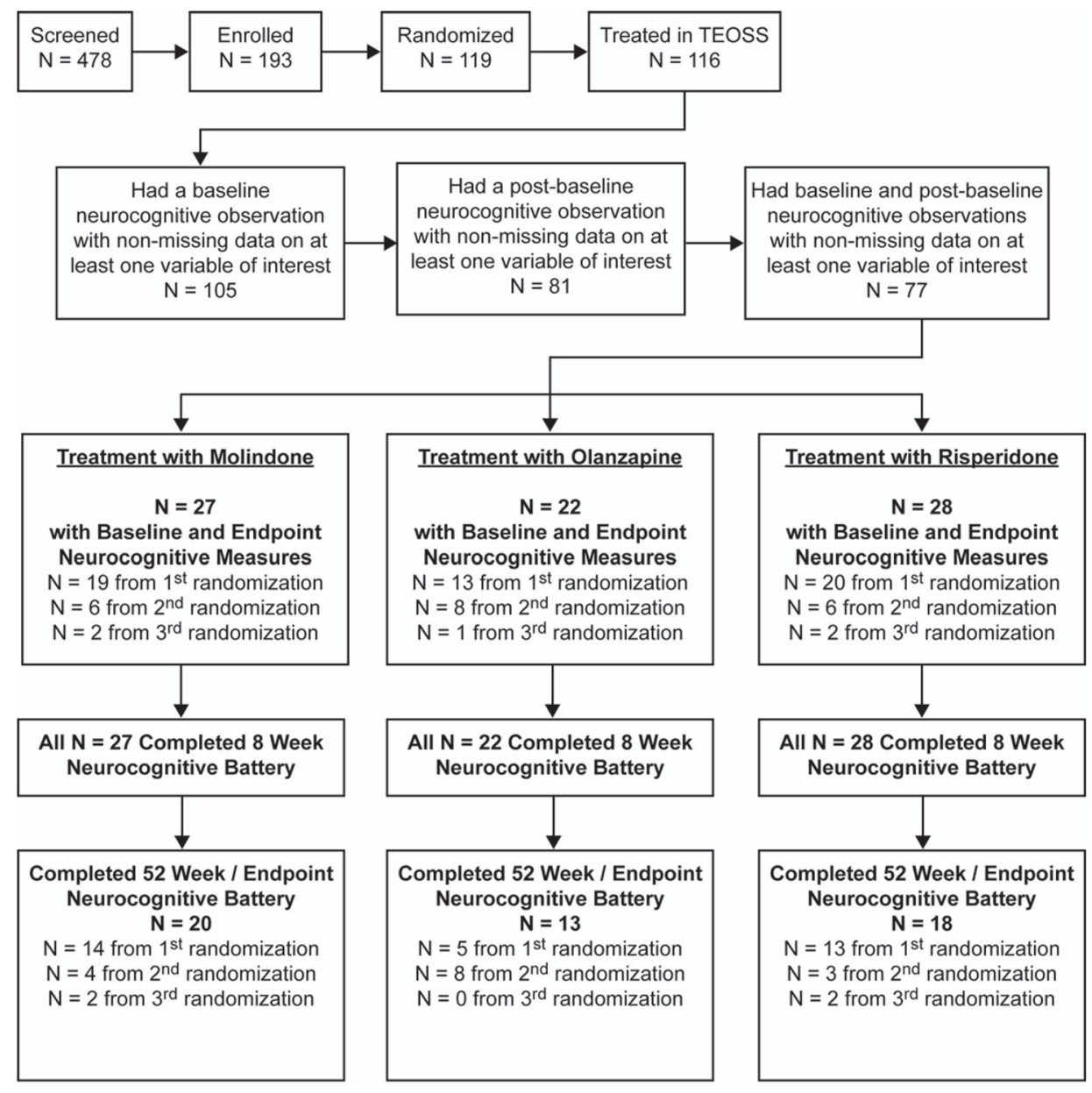

Figure 1.

Flow diagram of the progress through the Neurocognitive Battery phases of the Treatment of Early-Onset Schizophrenia Spectrum Disorders Study (TEOSS). 
Table 1

Treatment of Early-Onset Schizophrenia Spectrum Disorders (TEOSS) Neurocognitive Domain and Composite Scores

\begin{tabular}{|c|c|}
\hline Name of Domain/Composite & Measures \\
\hline \multicolumn{2}{|l|}{ Domain Scores } \\
\hline 1. Fine Motor Speed/Coordination & 1. Finger Tapping, Grooved Pegboard- dominant and non-dominant hand performance \\
\hline 2. Attention & 2. Auditory and visual VIGIL Continuous Performance Test (CPT) omission errors \\
\hline 3. Short-Term Memory & $\begin{array}{l}\text { 3. Hopkins Verbal Learning Test-Revised (HVLT-R) Total, Wide Range Assessment of Memory and } \\
\text { Learning (WRAML VL) Total, Visual Working Memory Test (VSWM) Mean Error 5-second delay, } \\
\text { Woodcock Johnson -III (WJ-III) Numbers Reversed Total }\end{array}$ \\
\hline 4. Cognitive Efficiency & $\begin{array}{l}\text { 4. Controlled Oral Word Association Test (COWAT) Letter and Semantic Fluency Total, Ruff } \\
\text { Figural Fluency Test (RFFT) Total Unique Designs }\end{array}$ \\
\hline 5. Inhibitory Control & 5. Auditory and visual VIGIL: CPT commission errors \\
\hline 6. Social Cognition & Eyes Test-Total Correct \\
\hline \multicolumn{2}{|l|}{ Composite Scores } \\
\hline 1. Academic Achievement & 1. Wide Range Achievement Test (WRAT-3) Reading, Spelling, Arithmetic Standard Scores \\
\hline 2. Full Scale IQ & 2. Four subtest Wechsler Abbreviated Scale of Intelligence (WASI) \\
\hline 3. Neurocognitive Composite Score & $\begin{array}{l}\text { 3. Unweighted average of Six Domain Scores: Fine Motor Speed/Coordination, Attention, Short- } \\
\text { Term Memory, Cognitive Efficiency, Inhibitory Control, and Social Cognition }\end{array}$ \\
\hline
\end{tabular}

Note: $\mathrm{CPT}=$ continuous performance test. 
Table 2

Baseline Demographic and Clinical Characteristics

\begin{tabular}{lll}
\hline Variable & Neurocognitive Sample N=77 & Non- Neurocognitive Sample N=39 \\
\hline Age (years) - Mean (SD) & $14.3(2.4)$ & $14.2(2.3)$ \\
Gender - \% Male & 66.2 & 61.5 \\
Race - \% White & 66.2 & 59.0 \\
Family income ${ }^{a}$ - Mean (SD) & $2.9(1.5)$ & $2.6(1.5)$ \\
Handedness - \% Right & 86.8 & 86.7 \\
Special Education - \% Yes & 57.1 & 51.3 \\
Diagnosis - \% Schizophrenia & 66.2 & 64.1 \\
\# prior psychiatric hospitalizations - Mean (SD) & $0.7(0.9)$ & $0.9(1.0)$ \\
Age at first psychosis - Mean (SD) & $11.0(3.5)$ & $11.3(3.4)$ \\
PANSS Total - Mean (SD) & $104.4(20.0)$ & ${ }^{*} 94.9(18.6)$ \\
PANSS Positive - Mean (SD) & $26.7(5.6)$ & $25.8(6.1)$ \\
PANSS Negative - Mean (SD) & $26.2(8.1)$ & ${ }^{*} 22.6(7.2)$ \\
CGI Severity - Mean (SD) & $5.7(0.8)$ & $5.4(0.9)$ \\
BPRS-C Total - Mean (SD) & $44.5(11.8)$ & $*^{*} 39.8(10.2)$ \\
\hline
\end{tabular}

Note: BPRS-C = Brief Psychiatric Scale for Children; CGI = Clinical Global Impression scale; PANSS = Positive and Negative Syndrome Scale

${ }^{a}$ Family income is described by the following categories: $1=<\$ 20 \mathrm{~K}, 2=\$ 20 \mathrm{~K}-<\$ 40 \mathrm{~K}, 3=\$ 40 \mathrm{~K}-<\$ 60 \mathrm{~K}, 4=\$ 60 \mathrm{~K}-<\$ 80 \mathrm{~K}, 5=\$ 80 \mathrm{~K}-<100 \mathrm{~K}$, and $6=$ $\$ 100 \mathrm{~K}+$.

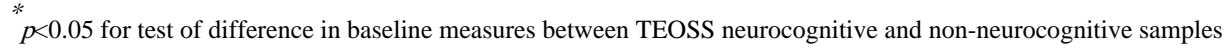




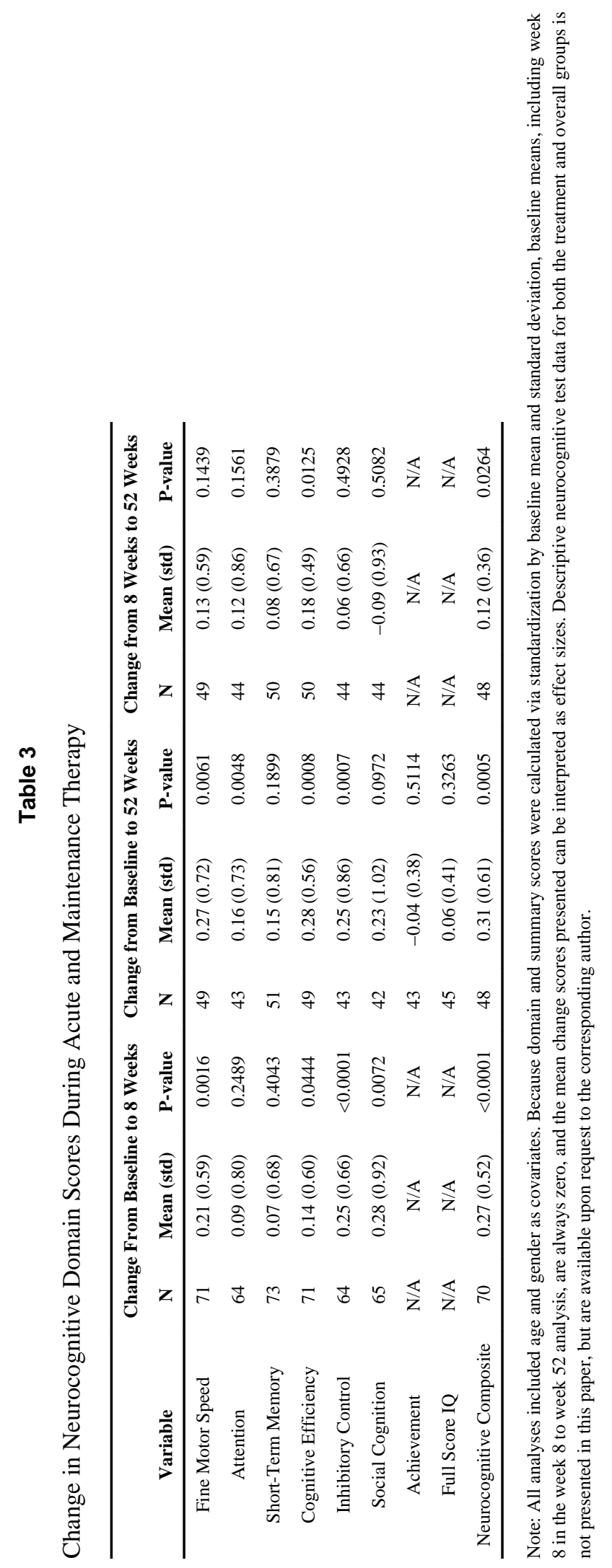

